\title{
EFECTOS DEL PARAQUAT SOBRE EL CRECIMIENTO Y LA MORFOLOGÍA DE LA MICROALGA Dunaliella tertiolecta.
}

\author{
Abelardo Calderón Rodríguez ${ }^{1}$ y Román Felipe Serpa Ibáñez ${ }^{2}$
}

\begin{abstract}
Resumen
Se estudió el efecto de diferentes dosis del herbicida metilviológeno paraquat, sobre el crecimiento y la morfología de la microalga Dunaliella tertiolecta bajo condiciones no controladas. Se encontró que la mejor forma de evaluar el crecimiento en este caso es la densidad celular. A las dosis menores de 0.008 ppm. se observó una tendencia creciente en la densidad celular, a dosis mayores hasta $0.04 \mathrm{ppm}$. la tendencia es decreciente. La forma y el movimiento se ven profundamente alterados a concentraciones altas, lo cual los lleva a la muerte aguda (no programada). En concentraciones bajas parece funcionar bien el sistema detoxificante SOD y carotenos, llegándose a determinar una dosis letal media de $0.006 \mathrm{ppm}$.
\end{abstract}

Palabras Clave: Dunaliella, paraquat, detoxificación.

\begin{abstract}
Different doses of the metilviologen herbicide paraquat were applied on the microalgae Dunaliella tertiolecta under non controlled conditions to determine the effects on the growth and the morphology. We found out that, the best way to evaluate the growth in this case is the cellular density. Doses lower than $0.008 \mathrm{ppm}$ had an increasing tendency in cellular density while greater doses up to $0.04 \mathrm{ppm}$ had a declining tendency. The morphology and the movement of the microalgae are profusely disturbed at high concentrations. Finally they die abruptly (non programmed death). At low concentrations, the detoxificant SOD and carotene system seem to function well. A lethal dose of $0.006 \mathrm{ppm}$ was found for this case.
\end{abstract}

Key words: Dunaliella, paraquat, detoxification.

\section{Introducción}

El paraquat (Dicloruro de 1,1-Dimetil4,4bipiridilo) es un reactivo que promueve la generación de radicales libres (FR) tales como el anión súperoxido $\mathrm{O}_{2}^{-}$y el altamente reactivo hidroxilo $\mathrm{OH}^{\cdot}$ que reacciona casi con cualquier clase de molécula orgánica. Estos radicales, conjuntamente con el peroxido de hidrógeno $\left(\mathrm{H}_{2} \mathrm{O}_{2}\right)$ son formas incompletamente reducidas del $\mathrm{O}_{2}$ y se conocen como especies de oxígeno reactivo (ROS) y tienen una alta reactividad aun bajo condiciones moderadas que facilitan la vida. Ellos oxidan a una gran variedad de biomoléculas. El ROS inactiva enzimas, oxida a los ácidos grasos insaturados de las membranas, y causa mella en los ácidos nucleicos con el riesgo de provocar mutaciones. A altas concentraciones, el ROS inicia la degradación de las proteínas, altera fuertemente la permeabilidad de las membranas celulares e induce la muerte celular programada o aguda. Estos efectos califican al paraquat como un herbicida de alta toxicidad (Dietz et al.,1998). En las plantas, el paraquat induce alteraciones en la ruta del transporte electrónico a nivel de reductor de la ferredoxina, aumentando el estrés oxidativo (Halliwel et al., 1987; Ascon - Bieto \& Talon, 1993).
Se ha reportado que el mecanismo de tolerancia implica elevados niveles de enzimas detoxificantes. Las enzimas antioxidantes mas prominentes son las ROS detoxificantes como la súperoxido dismutasa (SOD), $\mathrm{Cu} / \mathrm{Zn}-\mathrm{SOD}, \mathrm{Mn}-\mathrm{SOD}, \mathrm{Fe}-\mathrm{SOD}$, catalasas (CAT) y un gran numero de peroxidasas reductoras del $\mathrm{H}_{2} \mathrm{O}_{2}$. Otros antioxidantes no enzimáticos, como el glutation, el ascorbato, los carotenoídes, los flavonoides, el tocoferol, los ácidos ureíco y lipoíco ,varios aminoácidos ,poliaminas, y una variedad de compuestos fenólicos también cumplen un rol importante en la red de antioxidantes que protegen a las células ( Shannon et al., 1966; Larson, 1988).

Existen sin embargo estudios antagónicos (Kao \& Hassan, 1985; Bray et al., 1986) en los que haciendo uso de biotipos resistentes al paraquat no se ha encontrado incrementos en los niveles de enzimas detoxificantes. Por el contrario, se ha propuesto que la resistencia en estos biotipos se basa en un mecanismo de captura que evita que el paraquat alcance su sitio de acción en el cloroplasto o que se reduzca la translocación. El mecanismo de captura se da mas bien en organismos multicelulares; la tolerancia a la acción del paraquat a nivel de organismos unicelulares como E. coli y Chlorella sería mas bien atribuible a una disminución en la absorción del producto por

\footnotetext{
${ }^{1}$ Biólogo, M.Sc. Profesor Principal de Fisiología Vegetal. Departamento de Biología. Universidad Nacional Agraria La Molina, Lima - Perú. Apartado Postal 456 Lima 100 Perú. acalderon@lamolina.edu.pe

${ }_{2}^{2}$ Biólogo. Asistente de Investigación del Laboratorio de Fisiología Vegetal. Universidad Nacional Agraria La Molina, Lima - Perú.
} 
parte de las células antes que a niveles endógenos de enzimas detoxificantes. Esta sería una clase de resistencia al estrés iónico llamada evitación (Levitt, 1980).

El resultado del tratamiento con paraquat es un progresivo deterioro de la estructura celular (células hinchadas, citoplasma disgregado, alteración de la permeabilidad, las organelas no se hacen notorias y finalmente la muerte). Estudios ultra estructurales en algas unicelulares tales como la Chlorela y Clamydomonas (Bray et al., 1986) se han llevado a cabo en condiciones de laboratorio, pero no existen trabajos bajo condiciones no controladas, por tal motivo, el objetivo del presente trabajo, ha sido evaluar bajo condiciones no controladas de temperatura e irradiancia, el efecto del paraquat sobre la fisiología (crecimiento y motilidad) y ciertas características morfológicas del alga unicelular Dunaliella tertiolecta.

\section{Materiales y Métodos}

El experimento se realizó en un ambiente externo del Laboratorio de Fisiología Vegetal de la Universidad Nacional Agraria La Molina, durante fines de otoño $\mathrm{y}$ principios de primavera. La temperatura diurna varió entre los $20.8{ }^{\circ} \mathrm{C}$ y $22.9{ }^{\circ} \mathrm{C}$. El cielo se mostró totalmente cubierto durante los días que duró el experimento.

Se inoculó una alícuota de $5 \mathrm{ml}$. de una cepa de Dunaliella tertiolecta procedente del Instituto del Mar del Perú (IMARPE). a un litro de medio de cultivo agua de mar, envejecida, filtrada y enriquecida con las siguientes sales: $\mathrm{NO}_{3} \mathrm{Na}(75 \mathrm{mg}), \mathrm{NaH}_{2} \mathrm{PO}_{4} \mathrm{H}_{2} \mathrm{O}$ (5mg), $\mathrm{Na}_{2}$ EDTA (4.36mg), $\mathrm{Cl}_{3}$ Fe $6 \mathrm{H}_{2} \mathrm{O}$ (3.15mg), $\mathrm{SO}_{4} \mathrm{Cu} \quad 5 \mathrm{H}_{2} \mathrm{O} \quad(0.01 \mathrm{mg}), \mathrm{SO}_{4} \mathrm{Zn} \quad 7 \mathrm{H}_{2} \mathrm{O} \quad(0.022 \mathrm{mg})$, $\mathrm{Cl}_{2} \mathrm{Co} 6 \mathrm{H}_{2} \mathrm{O}(0.01 \mathrm{mg}), \mathrm{Cl}_{2} \mathrm{Mn} 4 \mathrm{H}_{2} \mathrm{O}(0.18 \mathrm{mg}), \mathrm{MoO}_{4}$ $\mathrm{Na}_{2} \quad 2 \mathrm{H}_{2} \mathrm{O} \quad(0.006 \mathrm{mg})$, Tiamina $\mathrm{HCl} \quad(0.1 \mathrm{mg})$, Biotina(0.5ug) y Vitamina B12 $(0.5 \mathrm{ug})$. El cultivo madre se mantuvo durante nueve horas bajo luz artificial.

\section{Prueba de Crecimiento control}

Se puso $10 \mathrm{ml}$. del medio de cultivo agua de mar enriquecida en cada uno de 20 viales. Estos medios fueron autoclavados durante 15 minutos a $120 \mathrm{C}$ y 15 libras de presión. Luego de enfriados fueron inoculados con $2 \mathrm{ml}$. del cultivo madre. La mitad de los viales se mantuvo en la cámara de crecimiento (laboratorio) y la otra mitad se cultivo en un ambiente externo del laboratorio.

El crecimiento en cada ambiente se determinó por medio de la densidad celular y por la absorbancia del cultivo a través del tiempo. Para cuantificar la densidad celular en cada vial, se traslado una alícuota de $0.9 \mathrm{ml}$ a otro vial y se le adicionó $0.1 \mathrm{ml}$. de lugol. De aquí se tomó una pequeña muestra para montarla en la cámara Newbauer y efectuar el conteo. En los casos de mucha densidad celular, la muestra se diluyó a razón de 1 en 10. Para cuantificar la absorbancia se usó la longitud de onda $640 \mathrm{~nm}$. que corresponde a la clorofila.

\section{Tratamiento con Paraquat}

Se prepararon 24 viales con $9 \mathrm{ml}$. de medio agua de mar enriquecido y esterilizado al autoclave a $120 \mathrm{C}$ durante 15 minutos. Los viales fueron luego inoculados con $1 \mathrm{ml}$. del cultivo madre y se incuban en la cámara de crecimiento. En los siguientes días se evalúa el crecimiento por absorbancia de clorofila. Después de seis días de crecimiento, 21 viales son agrupados de tres en tres para someterlos a las concentraciones siguientes del paraquat grado comercial; $(0.001,0.002,0.004,0.008,0.01,0.02$, $0.04 \mathrm{ppm})$, los 3 viales restantes sirvieron como controles.

Todos los cultivos fueron mantenidos en condiciones no controladas (exterior del laboratorio) la irradiancía promedio registrada fue de 1560 umol. $\mathrm{m}^{-2} \mathrm{~s}^{-1}$ con cielo nublado. Estos cultivos fueron analizados al día siguiente de haber recibido el tratamiento (séptimo día).

\section{Resultados y Discusión}

En la Figura 1 se observa que el crecimiento de Dunaliella tertiolecta es más pronunciado en el ambiente exterior donde la temperatura diurna varió entre $20.5^{\circ} \mathrm{C}$ y $23.5^{\circ} \mathrm{C}$ mientras que en condiciones del Laboratorio donde temperatura varió entre $18.25^{\circ} \mathrm{C}$ y $23^{\circ} \mathrm{C}$ es menor. Estas curvas se hicieron en función de la absorbancia de la clorofila (indicador de crecimiento), sin embargo no parece ser un indicador muy confiable, por cuanto, el cultivo desde la cepa madre al parecer no estuvo axénico. Una mejor forma de evaluación se presenta en la figura 2, en donde las curvas están en función de la densidad celular en el transcurrir de los días. En ella se observa una tendencia similar al encontrado con las absorbancias, a excepción de la disminución en la densidad en los últimos dos días que no se observa en el gráfico de absorbancias vs. días.

El análisis cuantitativo del crecimiento establece un intervalo de aproximadamente 10 a 11 días para llegar a un crecimiento máximo, lo cual corrobora lo encontrado por Aguilar (1995). Estas curvas, conjuntamente con otra estándar elaborada al momento del tratamiento con las dosis del paraquat, nos sirvieron para comparar los efectos a partir del sétimo día de crecimiento (primer día de tratamiento) hasta el día onceavo (sexto día de tratamiento)

De las Figuras 3.1 y 3.2 densidad celular vs. días (para cada dosis del paraquat) se observa que hay una tendencia al incremento en la densidad celular (crecimiento) en las dosis siguientes 0.001, 0.002, $0.004,0.008 \mathrm{ppm}$, mientras que existe una tendencia a la disminución en la densidad celular en las dosis $0.01,0.02,0.04 \mathrm{ppm}$. 


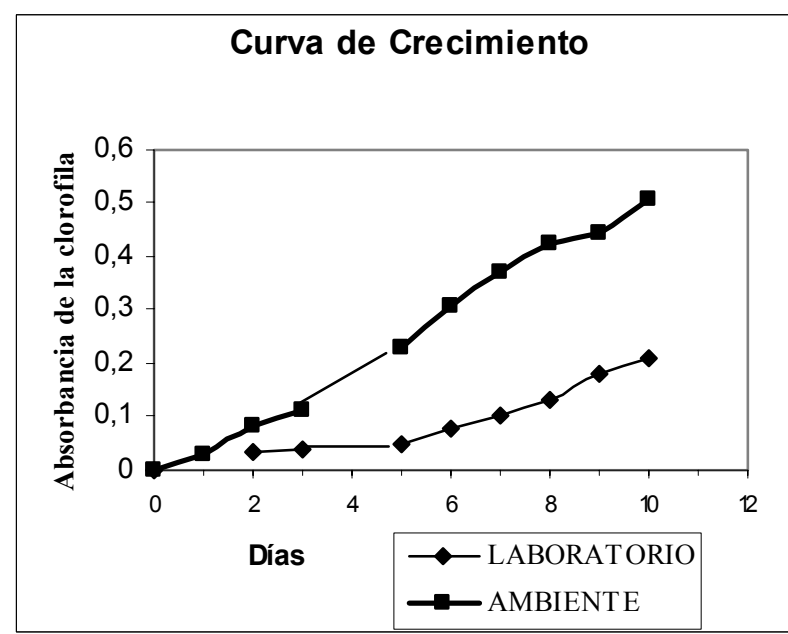

Figura 1. Evolución del crecimiento en función de la Absorción de la Clorofila a $(\lambda=680)$.

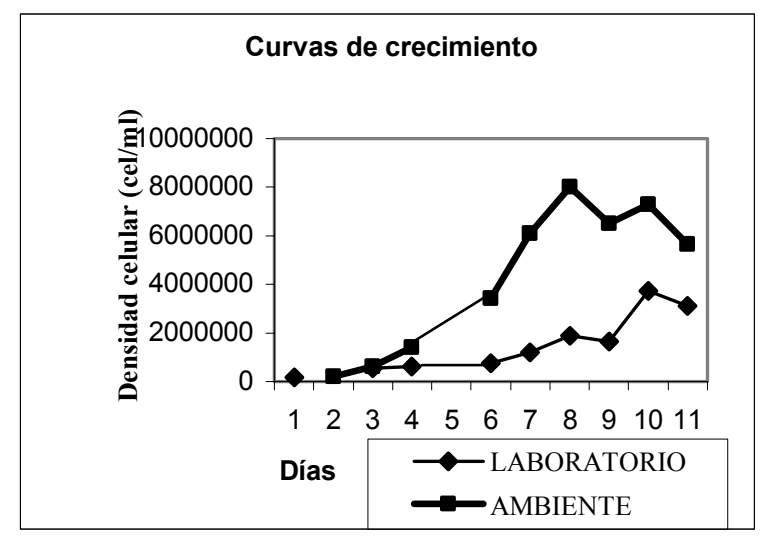

Figura 2. Evolución del crecimiento en función a la densidad celular en condiciones de laboratorio y ambiente natural.

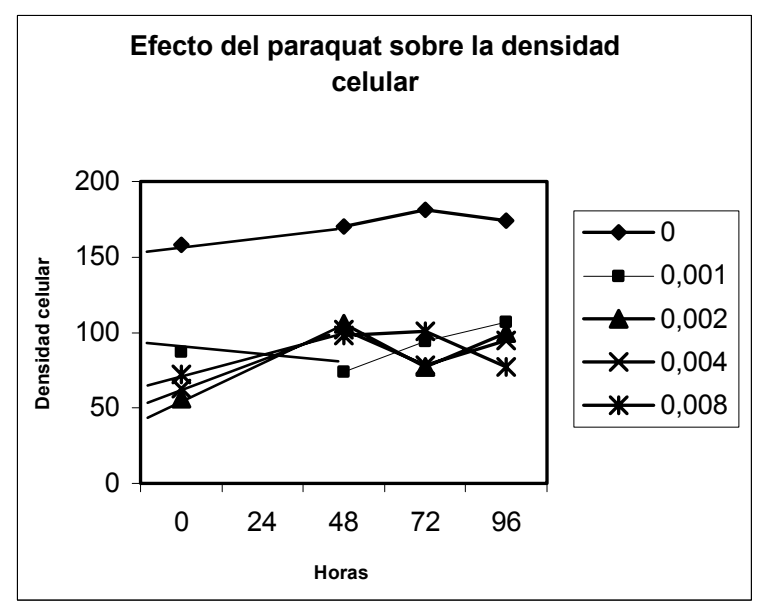

Figura 3.1. Efectos de niveles bajos de Paraquat sobre la Densidad celular en el lapso de 96 horas de exposición.

Para poder evaluar el grado de inhibición del crecimiento con respecto a un crecimiento normal, se graficó la relación porcentaje de sobrevivencia vs. dosis, Figura 4. Para hallar los valores de densidad celular se interpolaron los valores de absorbancia de los blancos mediante el uso de una curva estándar previamente elaborada; absorbancia vs. densidad celular. De esta forma, se observa una tendencia decreciente en el porcentaje de sobrevivencia al incrementar las dosis del paraquat y es probable que la dosis que represente un $50 \%$ de inhibición del crecimiento sea menor que $0.008 \mathrm{ppm}$ y mayor que $0.004 \mathrm{ppm}$.

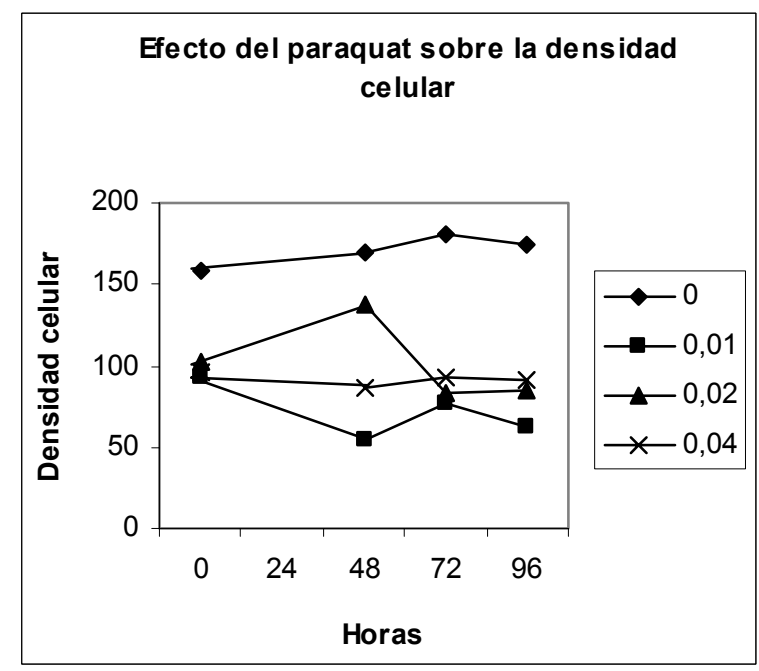

Figura 3.2. Efectos de niveles altos de Paraquat sobre la densidad celular en el lapso de 96 horas de exposición.

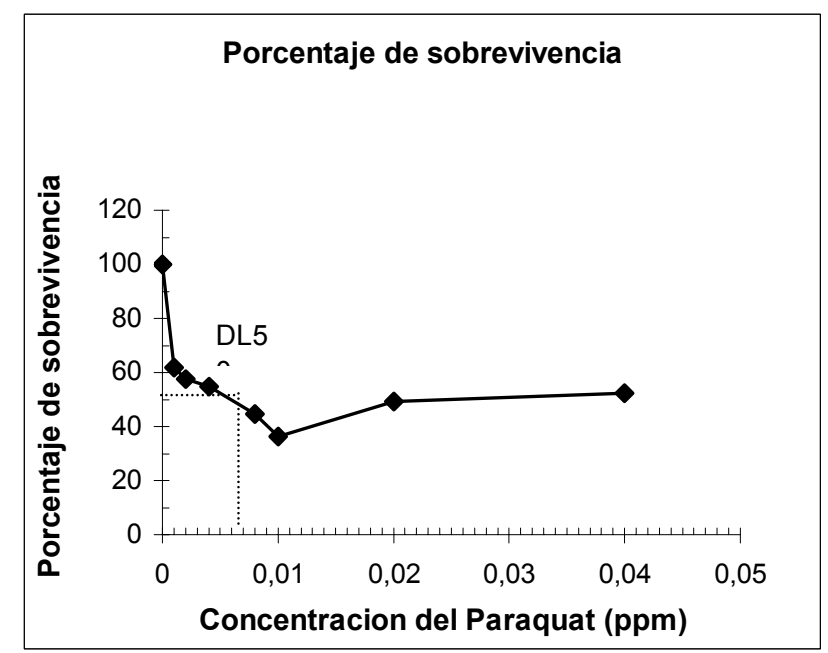

Figura 5. Determinación del Porcentaje de Sobrevivencia de Dunaliella tertiolecta a las distintos concentraciones del herbicida Paraquat.

De igual forma que en otros estudios (Bray et al., 1986), las células tratadas con paraquat en el presente trabajo, expresaron señales de deterioro solo después que estuvieron expuestas a la luz. Es más notorio el efecto, cuando se exponen a altas irradiancías como lo es la luz del microscopio usado para el conteo. En condiciones normales (Smith, 1951; Prescott, 1954; Lewis, 1962; Berner et al., 1989; Parra et al., 1990) el alga tiene una forma ovalada, con dos flagelos 
visibles, cloroplastos en forma de copa con un pirenoide en posición central penetrado por láminas tilakoidales, gránulos de almidón rodeando al pirenoide, un núcleo y tiene una tendencia a movilizarse hacia los bordes del cubreobjetos (fotofobia). Por otro lado, en un primer momento del tratamiento, en los niveles bajos se observa un movimiento normal, es decir rectilíneo, con un giro del alga sobre su eje dorsoventral, asemejando un tirabuzón; después de un cierto tiempo (aproximadamente media hora) se observa una agitación anómala (vibración) en algunas células. Al día siguiente, muchas células tienen un movimiento muy lento pero aun son ovoides, pero otros tantos están inmóviles y son esféricos, tal vez están muertos.

Normalmente, los cloroplastos y otros organelos producen niveles tolerables de oxígeno activo a través de la autooxidación del componente transportador de electrones del Fotosistema I. En la presencia de luz, el paraquat acelera la producción de superoxidos según Audus (1981). En niveles bajos del producto, el sistema antioxidante (SOD y otros) reforzado en este caso por la presencia del beta caroteno garantiza la sobrevivencia de los individuos más eficientes en su biosíntesis. Debemos anotar que Dunaliella tertiolecta es un buen productor de beta caroteno cuando se halla bajo estrés salino o de luz, siempre que la célula esté intacta. En niveles superiores al $0.008 \mathrm{ppm}$ los radicales libres (ROS) generan peroxidación de los lípidos de las membranas y por ello es que la permeabilidad se altera y entonces vemos células hinchadas o con incremento del volumen celular, tilakoides disgregados y una serie de daños morfológicos.

En las dosis mayores, los procesos descritos son similares pero más violentos y aparecen 15 minutos después de iniciado el tratamiento y a los 30 minutos la mayoría de las células están hinchadas e inmóviles. Las enzimas antioxidantes parecen no funcionar porque a nivel ultraestructural, todas las membranas dejan de ser funcionales, se pierde la compartimentación, se ven corrugadas, disgregadas, similares a los reportados para Chlamydomonas

Se sabe que la efectividad del herbicida en Chlorela está relacionada a la presencia en el medio de ciertos iones como el $\mathrm{Cu}(+2)$, que inhibe la actividad del paraquat a una concentración de 0.5 ppm. Audus (1981). El Cobre es cofactor de una enzima antioxidante $\mathrm{Cu} / \mathrm{Zn}$ SOD. Para Dunaliella no se han descrito mecanismos de resistencia, pero al parecer los carotenos juegan un rol importante hasta cierto punto.

\section{Literatura citada}

Aguilar C. 1995. Crecimiento y ciclo de vida de la microalga Dunaliella salina Teodoresco (Chlorophyta, Volvacales) de las salinas de Los
Chimus (Ancash) y las salinas de Chilca (Lima).Tesis para obtener el titulo de Licenciado en Biología. Universidad Ricardo Palma. LimaPerú.

Ascon - Bieto J. \& Talon M. 1993. Fisiología y Bioquímica Vegetal. Interamericana Mc Graw Hill -España.

Audus J. 1981. Herbicides. Academic Press, Inc., New York.

Ben-Amots A., Shaish A. \& Avron M. 1989. The mode of action of the massively accumulated betacarotene of Dunaliella bardawil in protecting the alga against the damage by excess irradiation. Plant Physiology. 9: 1040-1043.

Baier M. \& Dietz J. 1999. The Costs and Benefits of Oxygen for Photosynthesizing Plant Cells. Progress in Botany Vol 60. Springer - Verlag Berlin Heidelberg.

Berner T., Dubinski Z., Wyman K. \& Falkowski P. 1989. Photo adaptation and the package effect in Dunaliella tertiolecta. Journal Phicology. 25: 141146.

Bray D., Bagu J. \& Nakamura K. 1986. Ultra structure of Chlamydomonas reinhardtii following exposure to paraquat. Comparison of wild type and paraquat -resistant mutant. Canadian Journal Bot. 71: 174182 .

Dietz J., Baier M. \& Kramer U. 1998 Free Radicals and Reactive Oxygen Species as Mediators of Heavy Metal Toxicity in Plants. University of Bielefeld. D-33615. Germany.

Halliwell E. 1987. Oxidative damage, lipid peroxidation and antioxidant protection in Chloroplasts. Chemistry and Physics of lipids. 44: $327-340$

Kao S.M. \& Hassan H.M. 1985. Biochemical Characterization of a Paraquat - Tolerant Mutant of E coli. J. Biol. Chem. 260: 10478 - 10481.

Larson R.A. 1988. The antioxidants of higher plants. Phytochemistry. 27: $969-978$.

Levitt J. 1980. Responses of Plants to Environmental Stress. Academic Press, Inc New York Vol 1. Second Edition.

Lewis R.A. 1962 Physiology and Biochemistry of alga. Academic Press. New York - London.

Parra O., Gonzales M. \& De La Rosa V. 1990. Caracterización Biológica de una Cepa Chilena de Dunaliella salina Potencialmente Comerciable. Arch. Biol.Med.Exp. 23: 141 -146.

Prescott G.W. 1954. How to Know the Fresh Water Algae. Dubuque, Iowa, W.C Brown.

Shannon L., Kay E. \& Lewj Y. 1966. Peroxidase isozymes from horseradish roots. Isolation and Physical Properties. J.Biol Chem. 241: 2166-2172.

Smith G.M. 1951. Manual of Phycology. Waltham, Mass. Chronica Botanical. 